\title{
Dedekind sums with characters and class numbers of imaginary quadratic fields
}

\author{
by \\ KaORI OtA (Tokyo)
}

1. Introduction. In [4], we proved the reciprocity law for Dedekind sums with characters by using values at non-positive integers of Barnes's double zeta functions with characters. The reciprocity law (Theorem 1 in Section 2) is of course concerned with the Dedekind sums with characters. But if one is interested in the generalized Bernoulli numbers $B_{*, \chi}$ rather than in the Dedekind sums, then the law shows a different side. It leads us to consider a character as a product of two characters with coprime moduli if possible, and one might say that the law gives us the relation between $B_{*, \chi_{1} \chi_{2}}$ and $B_{*, \chi_{1}} \cdot B_{*, \chi_{2}}$, or that the Dedekind sums with characters fill the gap between $B_{*, \chi_{1} \chi_{2}}$ and $B_{*, \chi_{1}} \cdot B_{*, \chi_{2}}$.

From this point of view, we can obtain formulas for class numbers of imaginary quadratic fields, since $B_{1, \chi_{d}}$ for $\chi_{d}$ the Kronecker character is the negative of the class number of $\mathbb{Q}(\sqrt{d})$ when $d<-4$. More precisely, we prove three propositions (and one corollary). Actually the first two propositions are obtained by using the way of proving the law, and the last one is obtained from the law itself. Proposition 1 gives us a formula for the class number $h(d)$ of $\mathbb{Q}(\sqrt{d})$ by short character sums partitioned by $t$. We already have formulas for small values of $t$ (cf. [7, Chap. I, §9.6]), and Proposition 1 gives a general formula. Proposition 2 gives us an expression of $B_{1, \chi_{1} \chi_{2}}$ in terms of short character sums, where $\chi_{1}$ and $\chi_{2}$ are of opposite parity. This has been obtained by Szmidt, Urbanowicz and Zagier by using Zagier's identity (cf. [6], [7, Chap. I, §9.7]), and we give a different proof. The Corollary to Proposition 2 is known as the class number formulae of Lerch and Mordell (cf. [2], [3], [7, Chap. I, §9.7]). If we compare the formula for $h(t d)$ with that for $h(d)$, it may be possible to derive some interesting results (see Remark 1). The last proposition (Proposition 3) treats the relation between $h\left(d_{1}\right) h\left(d_{2}\right)$ and $h\left(\widetilde{d}_{1}\right) h\left(\widetilde{d}_{2}\right)$,

2000 Mathematics Subject Classification: 11R29, 11R11, 11F20, $11 \mathrm{~B} 68$. 
where $d_{1}, d_{2}, \widetilde{d}_{1}, \widetilde{d}_{2}<-4,\left(d_{1}, d_{2}\right)=\left(\widetilde{d}_{1}, \widetilde{d}_{2}\right)=1$ and $d_{1} d_{2}=\widetilde{d}_{1} \widetilde{d}_{2}$. They are related to the sums of the type $\sum_{n \in T} n \chi(n)$, where $\chi$ is the product of Kronecker characters for $\mathbb{Q}\left(\sqrt{d_{1}}\right)$ and $\mathbb{Q}\left(\sqrt{d_{2}}\right)$, and $T$ is the set $\left\{n=a\left|d_{1}\right|+b\left|d_{2}\right||a, b \in \mathbb{Z}, 0 \leq a<| d_{2}|, 0 \leq b<| d_{1} \mid, n>d_{1} d_{2}\right\}$. These sums come from the Dedekind sums.

In Section 2, we review Dedekind sums with a character, and state a reciprocity law for them. In Section 3, we give formulas for class numbers.

2. Dedekind sums with a character and the corresponding reciprocity law. First we give definitions of Bernoulli numbers, Bernoulli polynomials, Bernoulli functions and generalized Bernoulli numbers.

Definition 1. The $n$th Bernoulli number $B_{n}$ and the $n$th Bernoulli polynomial $B_{n}(u)$ are defined by

$$
\frac{t}{e^{t}-1}=\sum_{n=0}^{\infty} \frac{B_{n}}{n !} t^{n} \quad \text { and } \quad \frac{t e^{u t}}{e^{t}-1}=\sum_{n=0}^{\infty} \frac{B_{n}(u)}{n !} t^{n} .
$$

For example, $B_{1}=-1 / 2, B_{2}=1 / 6$ and $B_{1}(u)=u-1 / 2$. It is easy to show that

$$
B_{n}(1-u)=(-1)^{n} B_{n}(u) .
$$

The $n$th Bernoulli function $\bar{B}_{n}(u)$ is defined by

$$
\begin{aligned}
& \bar{B}_{n}(u)=B_{n}(\{u\}) \quad \text { if } n>1, \\
& \bar{B}_{1}(u)= \begin{cases}B_{1}(\{u\}) & \text { if } u \notin \mathbb{Z}, \\
0 & \text { if } u \in \mathbb{Z} .\end{cases}
\end{aligned}
$$

Here $\{u\}$ denotes the fractional part of a real number $u$, i.e., $0 \leq\{u\}<1$. Also for a Dirichlet character $\chi$ of conductor $f$, we define the $n$th generalized Bernoulli number $B_{n, \chi}$ by

$$
\sum_{a=1}^{f} \frac{\chi(a) t e^{a t}}{e^{f t}-1}=\sum_{n=0}^{\infty} \frac{B_{n, \chi}}{n !} t^{n} .
$$

It is easy to show that for any multiple $F$ of $f$,

$$
B_{n, \chi}=F^{n-1} \sum_{j=1}^{F} \chi(j) B_{n}\left(\frac{j}{F}\right) .
$$

The following gives us the definition of Dedekind sums with a character.

Definition 2. Let $k$ and $h$ be positive integers such that $(k, h)=1$, and let $\chi$ be a Dirichlet character defined $\bmod l$ with $l \mid k h$. We define the 
$n$th Dedekind sum with character $\chi$ by

$$
\begin{aligned}
& s_{n}(\chi ;(k, h))=k^{n-1} \sum_{a=0}^{k-1} \sum_{b=0}^{h-1} \frac{b}{h} \chi(h a+k b) \bar{B}_{n}\left(\frac{a}{k}+\frac{b}{h}\right), \\
& s_{n}(\chi ;(h, k))=h^{n-1} \sum_{a=0}^{k-1} \sum_{b=0}^{h-1} \frac{a}{k} \chi(h a+k b) \bar{B}_{n}\left(\frac{a}{k}+\frac{b}{h}\right) .
\end{aligned}
$$

For the principal character $\chi_{0}$, we have

$$
s_{n}\left(\chi_{0} ;(k, h)\right)=\sum_{b=1}^{h-1} \frac{b}{h} \bar{B}_{n}\left(\frac{k b}{h}\right)=: s_{n}(k, h),
$$

where $s_{n}(k, h)$ is the generalized Dedekind sum considered by Apostol (cf. [1]).

Lemma 1. (1) Let $\chi$ be a non-trivial character defined mod $k$, and set $\chi(-1)=(-1)^{\lambda}$. If $n \equiv \lambda(\bmod 2)$, then

$$
\left\{\begin{array}{l}
s_{n}(\chi ;(h, k))=\frac{1}{2} k^{1-n} B_{n, \chi}, \\
s_{n}(\chi ;(k, h))=\frac{1}{2}\left(h^{1-n}-\chi(h)\right) B_{n, \chi} .
\end{array}\right.
$$

(2) Let $\chi=\chi_{1} \chi_{2}$ be a character with $\chi_{1}\left(\right.$ resp. $\left.\chi_{2}\right)$ defined mod $k$ (resp. $h)$, both non-trivial, and set $\chi(-1)=(-1)^{\lambda}$. If $n \equiv \lambda(\bmod 2)$, then

$$
\left\{\begin{array}{l}
s_{n}(\chi ;(h, k))=\frac{1}{2} k^{1-n} B_{n, \chi}, \\
s_{n}(\chi ;(k, h))=\frac{1}{2} h^{1-n} B_{n, \chi} .
\end{array}\right.
$$

Proof. We only prove (1), since we can prove (2) analogously. Consider the sum

$$
A:=\sum_{a=0}^{k-1} \sum_{b=0}^{h-1} B_{1}\left(\frac{a}{k}\right) \chi(h a+k b) \bar{B}_{n}\left(\frac{a}{k}+\frac{b}{h}\right) .
$$

Since

$$
\left\{2-\left(\frac{a}{k}+\frac{b}{h}\right)\right\}= \begin{cases}1-\left(\frac{a}{k}+\frac{b}{h}\right) & \text { if } \frac{a}{k}+\frac{b}{h} \leq 1, \\ 2-\left(\frac{a}{k}+\frac{b}{h}\right) & \text { if } \frac{a}{k}+\frac{b}{h}>1,\end{cases}
$$

from (2.1) we have

$$
\begin{aligned}
\bar{B}_{n}(2- & \left.\left(\frac{a}{k}+\frac{b}{h}\right)\right) \\
= & \begin{cases}B_{n}\left(1-\left(\frac{a}{k}+\frac{b}{h}\right)\right)=(-1)^{n} \bar{B}_{n}\left(\frac{a}{k}+\frac{b}{h}\right) & \text { if } \frac{a}{k}+\frac{b}{h} \leq 1, \\
B_{n}\left(2-\left(\frac{a}{k}+\frac{b}{h}\right)\right)=(-1)^{n} \bar{B}_{n}\left(\frac{a}{k}+\frac{b}{h}\right) & \text { if } \frac{a}{k}+\frac{b}{h}>1 .\end{cases}
\end{aligned}
$$


So

$$
\begin{aligned}
A= & \sum_{a=1}^{k-1} \sum_{b=1}^{h-1} B_{1}\left(\frac{a}{k}\right) \chi(h a) \bar{B}_{n}\left(\frac{a}{k}+\frac{b}{h}\right)+\sum_{a=1}^{k-1} B_{1}\left(\frac{a}{k}\right) \chi(h a) \bar{B}_{n}\left(\frac{a}{k}\right) \\
= & \sum_{a=1}^{k-1} \sum_{b=1}^{h-1} B_{1}\left(\frac{k-a}{k}\right) \chi(h(k-a)) \bar{B}_{n}\left(\frac{k-a}{k}+\frac{h-b}{h}\right) \\
& +\sum_{a=1}^{k-1} B_{1}\left(\frac{k-a}{k}\right) \chi(h(k-a)) \bar{B}_{n}\left(\frac{k-a}{k}\right) \\
= & (-1)^{\lambda+n+1}\left\{\sum_{a=1}^{k-1} \sum_{b=1}^{h-1} B_{1}\left(\frac{a}{k}\right) \chi(h a) \bar{B}_{n}\left(\frac{a}{k}+\frac{b}{h}\right)\right. \\
& \left.+\sum_{a=1}^{k-1} B_{1}\left(\frac{a}{k}\right) \chi(h a) \bar{B}_{n}\left(\frac{a}{k}\right)\right\} \\
= & (-1)^{\lambda+n+1} A .
\end{aligned}
$$

Thus if $n \equiv \lambda(\bmod 2)$, then $A=0$.

Now since $B_{1}(u)=u-1 / 2$ and $\{h a+k b \mid 0 \leq a<k, 0 \leq b<h, a, b \in \mathbb{Z}\}$ is a complete set of representatives modulo $k h$, from (2.2) we have

$$
\begin{aligned}
s_{n}(\chi ;(h, k)) & =h^{n-1}\left\{A+\frac{1}{2} \sum_{a=0}^{k-1} \sum_{b=0}^{h-1} \chi(h a+k b) \bar{B}_{n}\left(\frac{a}{k}+\frac{b}{h}\right)\right\} \\
& =\frac{h^{n-1}}{2} \sum_{j=0}^{h k-1} \chi(j) B_{n}\left(\frac{j}{k h}\right)=\frac{1}{2} k^{1-n} B_{n, \chi}
\end{aligned}
$$

for $n \equiv \lambda(\bmod 2)$.

For $s_{n}(\chi ;(k, h))$, we obtain similarly

$$
\begin{aligned}
B & :=\sum_{a=0}^{k-1} \sum_{b=0}^{h-1} B_{1}\left(\frac{b}{h}\right) \chi(h a+k b) \bar{B}_{n}\left(\frac{a}{k}+\frac{b}{h}\right) \\
& =\sum_{a=1}^{k-1} \sum_{b=1}^{h-1} B_{1}\left(\frac{b}{h}\right) \chi(h a) \bar{B}_{n}\left(\frac{a}{k}+\frac{b}{h}\right)+B_{1}(0) \sum_{a=1}^{k-1} \chi(h a) \bar{B}_{n}\left(\frac{a}{k}\right) \\
& =(-1)^{\lambda+n+1} \sum_{a=1}^{k-1} \sum_{b=1}^{h-1} B_{1}\left(\frac{b}{h}\right) \chi(h a) \bar{B}\left(\frac{a}{k}+\frac{b}{h}\right)-\frac{1}{2} \chi(h) k^{1-n} B_{n, \chi} \\
& =(-1)^{\lambda+n+1}\left\{B+\frac{1}{2} \chi(h) k^{1-n} B_{n, \chi}\right\}-\frac{1}{2} \chi(h) k^{1-n} B_{n, \chi} .
\end{aligned}
$$


So if $n \equiv \lambda(\bmod 2)$, then

$$
B=-\frac{1}{2} \chi(h) k^{1-n} B_{n, \chi}
$$

Hence

$$
\begin{aligned}
s_{n}(\chi ;(k, h)) & =k^{n-1}\left\{B+\frac{1}{2} \sum_{a=0}^{k-1} \sum_{b=0}^{h-1} \chi(h a+k b) \bar{B}_{n}\left(\frac{a}{k}+\frac{b}{h}\right)\right\} \\
& =-\frac{1}{2} \chi(h) B_{n, \chi}+\frac{1}{2} h^{1-n} B_{n, \chi}=\frac{1}{2}\left(h^{1-n}-\chi(h)\right) B_{n, \chi} .
\end{aligned}
$$

The reciprocity law is the following:

TheOREM 1 (Reciprocity Law, [4]). Let $k, h$ and $\chi$ be as in Definition 2. If $\chi \neq \chi_{0}$, then for a positive integer $n$,

$$
\begin{aligned}
& \frac{1}{n}\left\{h^{n-1} s_{n}(\chi ;(k, h))+k^{n-1} s_{n}(\chi ;(h, k))\right\} \\
& \quad=\sum_{a=0}^{k-1} \sum_{b=0}^{h-1} \frac{\chi(h a+k b)(B h k+B h k+h a+k b)^{n+1}}{n(n+1)(h k)^{2}}+\frac{1}{h k} \frac{B_{n+1, \chi}}{n+1}+\frac{B_{n, \chi}}{n},
\end{aligned}
$$

where

$$
\begin{aligned}
& (B h k+B h k+h a+k b)^{n+1} \\
& =\sum_{j=0}^{n+1} \sum_{l=0}^{n+1-j}\left(\begin{array}{c}
n+1 \\
j
\end{array}\right)\left(\begin{array}{c}
n+1-j \\
l
\end{array}\right) B_{j}(h k)^{j} B_{l}(h k)^{l}(h a+k b)^{n+1-j-l} .
\end{aligned}
$$

For $n=1$ and $\chi=\chi_{1} \chi_{2}$ with $\chi_{1}\left(\right.$ resp. $\left.\chi_{2}\right)$ defined $\bmod k($ resp. $h)$, both non-trivial characters,

$$
s_{1}(\chi ;(k, h))+s_{1}(\chi ;(h, k))=u B_{1, \chi_{1}} B_{1, \chi_{2}}+\frac{B_{2, \chi}}{2 h k}+B_{1, \chi},
$$

where $u=\chi_{1}(h) \chi_{2}(k)$.

3. Formulas for class numbers of imaginary quadratic fields. In this section, we give several formulas for class numbers of imaginary quadratic fields. As usual, we denote the class number of a quadratic field $\mathbb{Q}(\sqrt{d})$ with a discriminant $d$ by $h(d)$.

Proposition 1. Let $d<-4$ be a discriminant of $\mathbb{Q}(\sqrt{d})$, and $t$ a positive integer such that $(t, d)=1$ and $t>1$. Then

$$
h(d)=\frac{1}{t-\chi_{d}(t)} \sum_{j=1}^{[t / 2]}(t-2 j+1) A_{j}\left(\chi_{d},|d|, t\right),
$$


where $\chi_{d}$ is the Kronecker character of $\mathbb{Q}(\sqrt{d})$, and $A_{j}\left(\chi_{d},|d|, t\right)$ is the short character sum defined by

$$
A_{j}\left(\chi_{d},|d|, t\right)=\sum_{|d|(j-1) / t \leq a<|d| j / t} \chi_{d}(a) .
$$

Proof. Set $d_{0}=|d|$ and $A_{j}=A_{j}\left(\chi_{d},|d|, t\right)$, and consider $B_{1, \chi_{d}}$. Since the set $\left\{a t+b d_{0} \mid 0 \leq a<d_{0}, 0 \leq b<t, a, b \in \mathbb{Z}\right\}$ is a complete set of representatives modulo $d_{0} t$, we have

$$
\begin{aligned}
B_{1, \chi_{d}} & =\sum_{j=0}^{d_{0} t-1} \chi_{d}(j) B_{1}\left(\frac{j}{d_{0} t}\right)=\sum_{a=0}^{d_{0}-1} \sum_{b=0}^{t-1} \chi_{d}\left(a t+b d_{0}\right) \bar{B}_{1}\left(\frac{a t+b d_{0}}{d_{0} t}\right) \\
& =\sum_{a=0}^{d_{0}-1} \sum_{b=0}^{t-1} \chi_{d}(a t)\left(\left\{\frac{a}{d_{0}}+\frac{b}{t}\right\}-\frac{1}{2}\right) \\
& =\sum_{a=0}^{d_{0}-1} \sum_{b=0}^{t-1} \chi_{d}(a t)\left(\frac{a}{d_{0}}+\frac{b}{t}-\frac{1}{2}\right)-\sum_{(a, b) \in S} \chi_{d}(a t),
\end{aligned}
$$

where

$$
S=\left\{(a, b) \in \mathbb{Z}^{2} \mid 0 \leq a<d_{0}, 0 \leq b<t, a t+b d_{0} \geq d_{0} t\right\}
$$

Now

$$
\sum_{a=0}^{d_{0}-1} \sum_{b=0}^{t-1} \chi_{d}(a t)\left(\frac{a}{d_{0}}+\frac{b}{t}-\frac{1}{2}\right)=\chi_{d}(t) t \sum_{a=1}^{d_{0}-1} \frac{a}{d_{0}} \chi_{d}(a)=\chi_{d}(t) t B_{1, \chi_{d}}
$$

Hence from (3.1) and (3.2) we have

$$
B_{1, \chi_{d}}=\frac{1}{t-\chi_{d}(t)} \sum_{(a, b) \in S} \chi_{d}(a) .
$$

Since $(a, b) \in S$ if and only if $a \geq d_{0}-b d_{0} / t$

$$
\begin{aligned}
\sum_{(a, b) \in S} \chi_{d}(a) & =\sum_{b=1}^{t-1} \sum_{a \geq d_{0}-b d_{0} / t} \chi_{d}(a) \\
& =-\sum_{b=1}^{t-1} \sum_{a<b d_{0} / t} \chi_{d}(a)=-\sum_{b=1}^{t-1} \sum_{j=1}^{b} A_{j} \\
& =-\sum_{j=1}^{t-1}\left(\sum_{b=j}^{t-1} 1\right) A_{j}=-\sum_{j=1}^{t-1}(t-j) A_{j} .
\end{aligned}
$$


Assume that $t$ is odd. Then

$$
\begin{aligned}
\sum_{j=1}^{t-1}(t-j) A_{j} & =\sum_{j=1}^{(t-1) / 2}(t-j) A_{j}+\sum_{j=(t+1) / 2}^{t-1}(t-j) A_{j} \\
& =\sum_{j=1}^{(t-1) / 2}(t-j) A_{j}+\sum_{j=1}^{(t-1) / 2} j A_{t-j} .
\end{aligned}
$$

Since

$$
\begin{aligned}
A_{t-j} & =\sum_{|d|(t-j-1) / t \leq a<|d|(t-j) / t} \chi_{d}(a) \\
& =\sum_{|d| j / t<b \leq|d|(j+1) / t} \chi_{d}(|d|-b)=-A_{j+1},
\end{aligned}
$$

we have $A_{(t+1) / 2}=0$. Thus

$$
\sum_{j=1}^{t-1}(t-j) A_{j}=\sum_{j=1}^{(t-1) / 2}(t-2 j+1) A_{j} .
$$

For $t$ even we can show analogously

$$
\sum_{j=1}^{t-1}(t-j) A_{j}=\sum_{j=1}^{t / 2}(t-2 j+1) A_{j} .
$$

Therefore from (3.3), (3.4), (3.6) and (3.7), we get

$$
B_{1, \chi_{d}}=\frac{-1}{t-\chi_{d}(t)} \sum_{j=1}^{[t / 2]}(t-2 j+1) A_{j} .
$$

As $h(d)=-B_{1, \chi_{d}}$ for $d<-4$, we have the result.

EXAMPLE. For $t=2,3,5$, the formula gives us known results:

$$
\begin{aligned}
& h(d)=\frac{1}{2-\chi_{d}(2)} \sum_{a<|d| / 2} \chi_{d}(a), \quad h(d)=\frac{2}{3-\chi_{d}(3)} \sum_{a<|d| / 3} \chi_{d}(a), \\
& h(d)=\frac{2}{5-\chi_{d}(5)}\left\{2 \sum_{a<|d| / 5} \chi_{d}(a)+\sum_{|d| / 5<a<2|d| / 5} \chi_{d}(a)\right\}
\end{aligned}
$$

(cf. [7, Chap. I, §9.6]). For $t=7$,

$$
\begin{aligned}
h(d)= & \frac{2}{7-\chi_{d}(7)} \\
& \times\left\{3 \sum_{a<|d| / 7} \chi_{d}(a)+2 \sum_{|d| / 7<a<2|d| / 7} \chi_{d}(a)+\sum_{2|d| / 7<a<3|d| / 7} \chi_{d}(a)\right\}
\end{aligned}
$$

for $d$ with $(d, 7)=1$. 
Next we shall consider $t$ such that $t$ is also a discriminant of $\mathbb{Q}(\sqrt{t})$ and $(t, d)=1$. Then $t d$ is a discriminant of $\mathbb{Q}(\sqrt{t d})$. The following proposition is essentially the same as Theorem 7 in [7, Chap. I], which was proved by using Zagier's identity. We give a different proof.

Proposition 2 ([6], [7, Chap. I, Theorem 7]). Let $k$ and $h$ be positive integers with $(k, h)=1$, and $\chi_{1}$ and $\chi_{2}$ non-trivial Dirichlet characters defined $\bmod k$ and $h$ respectively, satisfying $\chi_{1}(-1)=-1$ and $\chi_{2}(-1)=1$. Then

$$
\begin{aligned}
B_{1, \chi_{1} \chi_{2}} & =-2 \chi_{1}(h) \chi_{2}(k) \sum_{j=1}^{[h / 2]}\left(\sum_{l=1}^{j-1} \chi_{2}(l)\right) A_{j}\left(\chi_{1}, k, h\right) \\
& =2 \chi_{1}(h) \chi_{2}(k) \sum_{j=1}^{[k / 2]}\left(\sum_{l=1}^{j-1} \chi_{1}(l)\right) A_{j}\left(\chi_{2}, h, k\right)+\alpha
\end{aligned}
$$

where

$$
A_{j}\left(\chi_{1}, k, h\right)=\sum_{k(j-1) / h \leq a<k j / h} \chi_{1}(a), \quad A_{j}\left(\chi_{2}, h, k\right)=\sum_{h(j-1) / k \leq a<h j / k} \chi_{2}(a)
$$

and

$$
\alpha= \begin{cases}\chi_{1}(h) \chi_{2}(k)\left(\sum_{l=1}^{(k-1) / 2} \chi_{1}(l)\right) A_{(k+1) / 2}\left(\chi_{2}, h, k\right) & \text { for } k \text { odd }, \\ 0 & \text { for } k \text { even } .\end{cases}
$$

Proof. Similarly to the proof of Proposition 1, we have

$$
\begin{aligned}
B_{1, \chi_{1} \chi_{2}} & =\sum_{j=0}^{k h-1} \chi_{1} \chi_{2}(j) B_{1}\left(\frac{j}{k h}\right)=\sum_{a=0}^{k-1} \sum_{b=0}^{h-1} \chi_{1} \chi_{2}(h a+k b) \bar{B}_{1}\left(\frac{h a+k b}{k h}\right) \\
& =\sum_{a=0}^{k-1} \sum_{b=0}^{h-1} \chi_{1}(h a) \chi_{2}(k b)\left(\frac{a}{k}+\frac{b}{h}-\frac{1}{2}\right)-\sum_{(a, b) \in S_{1}} \chi_{1}(h a) \chi_{2}(k b),
\end{aligned}
$$

where

$$
S_{1}=\left\{(a, b) \in \mathbb{Z}^{2} \mid 0 \leq a<k, 0 \leq b<h, h a+k b \geq k h\right\}
$$

Now

$$
\sum_{a=0}^{k-1} \sum_{b=0}^{h-1} \chi_{1}(h a) \chi_{2}(k b)\left(\frac{a}{k}+\frac{b}{h}-\frac{1}{2}\right)=0,
$$

since $\chi_{1}$ and $\chi_{2}$ are both non-trivial. Thus

$$
B_{1, \chi_{1} \chi_{2}}=-\chi_{1}(h) \chi_{2}(k) \sum_{(a, b) \in S_{1}} \chi_{1}(a) \chi_{2}(b) .
$$


As in the proof of Proposition 1,

$$
\begin{aligned}
\sum_{(a, b) \in S_{1}} \chi_{1}(a) \chi_{2}(b) & =\sum_{b=1}^{h-1} \chi_{2}(b) \sum_{a \geq k-k b / h} \chi_{1}(a) \\
& =-\sum_{b=1}^{h-1} \chi_{2}(b) \sum_{a<k b / h} \chi_{1}(a) \\
& =-\sum_{b=1}^{h-1} \chi_{2}(b) \sum_{j=1}^{b} A_{j}\left(\chi_{1}, k, h\right) \\
& =-\sum_{j=1}^{h-1}\left(\sum_{b=j}^{t-1} \chi_{2}(b)\right) A_{j}\left(\chi_{1}, k, h\right) \\
& =\sum_{j=1}^{h-1}\left(\sum_{b=1}^{j-1} \chi_{2}(b)\right) A_{j}\left(\chi_{1}, k, h\right) .
\end{aligned}
$$

By using $A_{h-j}\left(\chi_{1}, k, h\right)=-A_{j+1}\left(\chi_{1}, k, h\right)$ (cf. (3.5)), we derive

$$
\sum_{j=1}^{h-1}\left(\sum_{b=1}^{j-1} \chi_{2}(b)\right) A_{j}\left(\chi_{1}, k, h\right)=2 \sum_{j=1}^{[h / 2]}\left(\sum_{b=1}^{j-1} \chi_{2}(b)\right) A_{j}\left(\chi_{1}, k, h\right) .
$$

Therefore from (3.10)-(3.12), we obtain (3.8).

For (3.9), we do the same computations except that we use the identity

$$
A_{k-j}\left(\chi_{2}, h, k\right)=A_{j+1}\left(\chi_{2}, h, k\right) .
$$

Corollary (Class number formulae of Lerch and Mordell, [2], [3], [7, Chap. I, Theorem 6]). Let $d$ be a discriminant of an imaginary quadratic field $\mathbb{Q}(\sqrt{d})$, and $t$ a discriminant of a real quadratic field $\mathbb{Q}(\sqrt{t})$ satisfying $(t, d)=1$. Then

$$
h(t d)=2 \sum_{j=1}^{[t / 2]}\left(\sum_{l=1}^{j-1} \chi_{t}(l)\right) A_{j}\left(\chi_{d},|d|, t\right),
$$

where $A_{j}\left(\chi_{d},|d|, t\right)$ is the same as in Proposition 1.

Proof. We only note that under our assumptions $\chi_{d}(t) \chi_{t}(|d|)=1$.

REMARK 1. If we compare the above Corollary with Proposition 1, we might obtain some interesting results. For example, when $t$ is a prime with $t \equiv 1(\bmod 4)$, the Corollary gives us the congruence

$$
h(t d) \equiv\left(1-\chi_{d}(t)\right) h(d)(\bmod 4) .
$$


This is because

$$
\begin{aligned}
h(t d) & =2 \sum_{j=2}^{(t-1) / 2}\left\{\sum_{l=1}^{j-1}\left(\left(\frac{l}{t}\right)+1\right)\right\} A_{j}-2 \sum_{j=2}^{(t-1) / 2}\left(\sum_{l=1}^{j-1} 1\right) A_{j} \\
& \equiv 2 \sum_{j=2}^{(t-1) / 2}(1-j) A_{j} \equiv\left(t-\chi_{d}(t)\right) h(d) \equiv\left(1-\chi_{d}(t)\right) h(d)(\bmod 4) .
\end{aligned}
$$

The above congruence is obvious if $h(d)$ is even. But if $h(d)$ is odd, i.e., the 2-rank of the ideal class group of $\mathbb{Q}(\sqrt{t d})$ is 1 , then

$$
h(t d) \equiv 0(\bmod 4) \Leftrightarrow \chi_{d}(t)=1,
$$

which is a part of the results by Rédei and Reichardt (cf. [5]).

Next we shall consider the relation between $h\left(d_{1}\right) h\left(d_{2}\right)$ and $h\left(\widetilde{d}_{1}\right) h\left(\widetilde{d}_{2}\right)$, where $d_{1}, d_{2}, \widetilde{d}_{1}, \widetilde{d}_{2}$ are discriminants of imaginary quadratic fields such that $\left(d_{1}, d_{2}\right)=\left(\widetilde{d}_{1}, \widetilde{d}_{2}\right)=1$ and $d_{1} d_{2}=\widetilde{d}_{1} \widetilde{d}_{2}$.

We define the Dirichlet $L$-series $L(s, \chi)$ by

$$
L(s, \chi)=\sum_{n=1}^{\infty} \frac{\chi(n)}{n^{s}} \quad \text { for } \operatorname{Re}(s)>1 .
$$

It is well known that $L(s, \chi)$ is analytically continued to the whole complex plane, and for a positive integer $n$,

We also set

$$
L(1-n, \chi)=-\frac{B_{n, \chi}}{n} .
$$

$$
T(k, h)=\{n=h a+k b \mid a, b \in \mathbb{Z}, 0 \leq a<k, 0 \leq b<h, n>k h\}
$$

for coprime positive integers $k$ and $h$.

LEMma 2. Let $\chi_{1}$ and $\chi_{2}$ be non-trivial characters defined mod $k$ and $h$, respectively. Assume $(k, h)=1$, and set $\chi=\chi_{1} \chi_{2}$.

(1) $L(0, \chi)=\sum_{n \in T(k, h)} \chi(n)$.

(2) When $\chi_{1}$ and $\chi_{2}$ are both even,

$$
L(-1, \chi)=\sum_{n \in T(k, h)} n \chi(n) .
$$

(3) When $\chi_{1}$ and $\chi_{2}$ are both odd,

$$
L(-1, \chi)=-\chi_{1}(h) \chi_{2}(k) k h B_{1, \chi_{1}} B_{1, \chi_{2}}+\sum_{n \in T(k, h)} n \chi(n) .
$$

(4) When $\chi_{1}$ and $\chi_{2}$ are of opposite parity,

$$
L(0, \chi)=\frac{1}{k h} \sum_{n \in T(k, h)} n \chi(n) .
$$


Proof. For (1), we argue as follows:

$$
\begin{aligned}
B_{1, \chi} & =\sum_{a=0}^{k-1} \sum_{b=0}^{h-1} \chi(h a+k b) \bar{B}_{1}\left(\frac{a}{k}+\frac{b}{h}\right) \\
& =\sum_{a=0}^{k-1} \sum_{b=0}^{h-1} \chi(h a+k b)\left(\frac{a}{k}+\frac{b}{h}-\frac{1}{2}\right)-\sum_{n \in T(k, h)} \chi(n) \\
& =-\sum_{n \in T(k, h)} \chi(n) .
\end{aligned}
$$

For (2)-(4), we apply Theorem 1.

(2) The right hand side of $(2.3)$ is $-\frac{1}{k h} L(-1, \chi)$, and the left hand side of $(2.3)$ is

$$
\chi_{1}(h) \chi_{2}(k) \sum_{a=0}^{k-1} \sum_{b=0}^{h-1}\left(\frac{a}{k}+\frac{b}{h}\right) \chi_{1}(a) \chi_{2}(b)\left(\frac{a}{k}+\frac{b}{h}-\frac{1}{2}\right)-\frac{1}{k h} \sum_{n \in T(k, h)} n \chi(n) .
$$

Here the first term becomes 0 since both are even non-trivial characters, and we obtain the result.

(3) Similarly the right hand side of (2.3) is

$$
-\frac{1}{k h} L(-1, \chi)+\chi_{1}(h) \chi_{2}(k) B_{1, \chi_{1}} B_{1, \chi_{2}},
$$

and the left hand side is

$$
2 \chi_{1}(h) \chi_{2}(k) B_{1, \chi_{1}} B_{1, \chi_{2}}-\frac{1}{k h} \sum_{n \in T(k, h)} n \chi(n) .
$$

(4) The right hand side of $(2.3)$ is $-L(0, \chi)$, and the left hand side is $-\frac{1}{k h} \sum_{n \in T(k, h)} n \chi(n)$.

The following proposition is an easy consequence of the above lemma.

Proposition 3. (1) Let $d$ be a discriminant of an imaginary quadratic field, and $\chi_{d}$ the Kronecker character. Suppose that we have a decomposition of $|d|$ as $|d|=k h$ with $k, h>1$ and $(k, h)=1$. Then

$$
h(d)=\sum_{n \in T(k, h)} \chi_{d}(n) .
$$

(2) Let $d_{1}$ and $d_{2}$ be discriminants of imaginary quadratic fields with

$$
\left(d_{1}, d_{2}\right)=1 \quad \text { and } \quad d_{1}, d_{2}<-4 .
$$

Also let $\chi_{i}$ be the corresponding Kronecker character, and set $\chi=\chi_{1} \chi_{2}$. 
(a) We have the formula

$$
h\left(d_{1}\right) h\left(d_{2}\right)=\frac{1}{d_{1} d_{2}} L(-1, \chi)-\frac{1}{d_{1} d_{2}} \sum_{n \in T\left(\left|d_{1}\right|,\left|d_{2}\right|\right)} n \chi(n) .
$$

(b) When $d_{1} \equiv 1(\bmod 4)$ and $d_{2}=4 m_{2}$ with $m_{2} \equiv 3(\bmod 4)$,

$$
h\left(d_{1}\right) h\left(d_{2}\right)=\frac{1}{d_{1} d_{2}}\left\{\sum_{n \in T\left(4\left|d_{1}\right|,\left|m_{2}\right|\right)} n \chi(n)-\sum_{n \in T\left(\left|d_{1}\right|,\left|d_{2}\right|\right)} n \chi(n)\right\} .
$$

(c) Suppose that we have another pair $\left(\widetilde{d}_{1}, \widetilde{d}_{2}\right)$ of discriminants for imaginary quadratic fields satisfying (3.13) and $d_{1} d_{2}=\widetilde{d}_{1} \widetilde{d}_{2}$. Then

$$
h\left(d_{1}\right) h\left(d_{2}\right)-h\left(\widetilde{d}_{1}\right) h\left(\widetilde{d}_{2}\right)=\frac{1}{d_{1} d_{2}}\left\{\sum_{n \in T\left(\left|\widetilde{d}_{1}\right|,\left|\widetilde{d}_{2}\right|\right)} n \chi(n)-\sum_{n \in T\left(\left|d_{1}\right|,\left|d_{2}\right|\right)} n \chi(n)\right\} .
$$

Proof. (1) Corresponding to $|d|=k h$, we have the decomposition of $\chi_{d}$ as $\chi_{d}=\chi_{1} \chi_{2}$ with $\chi_{1}$ and $\chi_{2}$ defined $\bmod k$ and $h$, respectively. So from Lemma 2(1) this is obvious.

(2) First we note that $\chi_{1}\left(\left|d_{2}\right|\right) \chi_{2}\left(\left|d_{1}\right|\right)=-1$.

(a) This is obvious from Lemma $2(3)$.

(b) We have $d_{1} d_{2}=\left(-4 d_{1}\right)\left(-m_{2}\right)$, where $-4 d_{1}$ and $-m_{2}$ are discriminants of real quadratic fields, and the product of their Kronecker characters is the same as $\chi=\chi_{1} \chi_{2}$. From Lemma 2(2) we know that

$$
L(-1, \chi)=\sum_{n \in T\left(4\left|d_{1}\right|,\left|m_{2}\right|\right)} n \chi(n) .
$$

On the other hand, from Lemma 2(3) we have another expression of $L(-1, \chi)$, and by equating the two the result is obtained.

(c) Since $\chi_{1} \chi_{2}=\widetilde{\chi}_{1} \widetilde{\chi}_{2}$, this is obvious from (a).

Remark 2. (1) When $d_{1}$ (or $d_{2}$ ) in Proposition $3(2)$ is -3 or -4 , the results are slightly different, as $h\left(d_{1}\right)=-3 B_{1, \chi_{1}}$ or $-2 B_{1, \chi_{1}}$.

(2) It seems meaningful to consider the values $\sum_{n \in T} n \chi(n)$ for various $T$ 's. When $\chi=\chi_{0}$, the principal character, we can evaluate the sum:

$$
\sum_{n \in T(k, h)} n=\frac{2}{3}(k h)^{2}-\frac{3}{4} k h(k+h)+\frac{7}{12} k h+\frac{1}{12}(k+h)^{2}-\frac{1}{12} .
$$

This is obtained for example by using the reciprocity law of Dedekind:

$$
s_{1}(k, h)+s_{1}(h, k)=\frac{1}{12}\left(\frac{k}{h}+\frac{h}{k}+\frac{1}{k h}\right)-\frac{1}{4} .
$$


For we can transform the left hand side of the law as follows:

$$
\begin{aligned}
s_{1}(k, h)+s_{1}(h, k) & =\sum_{b=1}^{h-1} \frac{b}{h} \bar{B}_{1}\left(\frac{k b}{h}\right)+\sum_{a=1}^{k-1} \frac{a}{k} \bar{B}_{1}\left(\frac{h a}{k}\right) \\
& =\sum_{b=1}^{h-1} \frac{b}{h} \sum_{a=0}^{k-1} \bar{B}_{1}\left(\frac{b}{h}+\frac{a}{k}\right)+\sum_{a=1}^{k-1} \frac{a}{k} \sum_{b=0}^{h-1} \bar{B}_{1}\left(\frac{a}{k}+\frac{b}{h}\right) \\
& =\sum_{a=0}^{k-1} \sum_{b=0}^{h-1}\left(\frac{a}{k}+\frac{b}{h}\right) \bar{B}_{1}\left(\frac{a}{k}+\frac{b}{h}\right) \\
& =\sum_{a=0}^{k-1} \sum_{b=0}^{h-1}\left(\frac{a}{k}+\frac{b}{h}\right)\left(\frac{a}{k}+\frac{b}{h}-\frac{1}{2}\right)-\frac{1}{k h} \sum_{n \in T(k, h)} n .
\end{aligned}
$$

$\sum_{n \in T(k, h)} n \chi(n)$ is considered as the signed sum over the set $T(k, h)$.

Acknowledgements. The author would like to thank Hirotada Naito for pointing out the results of Rédei and Reichardt.

\section{References}

[1] T. Apostol, Generalized Dedekind sums and transformation formulae of certain Lambert series, Duke Math. J. 17 (1950), 147-157.

[2] M. Lerch, Essai sur le calcul du nombre de classes de formes quadratiques binaires aux coefficients entiers, Acta Math. 29 (1905), 333-424.

[3] L. J. Mordell, On Lerch's class number for binary quadratic forms, Ark. Mat. 5 (1963), 97-100.

[4] Y. Nagasaka, K. Ota and C. Sekine, Generalizations of Dedekind sums and their reciprocity laws, Acta Arith. 106 (2003), 355-378.

[5] L. Rédei und H. Reichardt, Die Anzahl der durch 4 teilbaren Invarianten der Klassengruppe eines beliebigen quadratischen Zahlkörpers, J. Reine Angew. Math. 170 (1933), 69-74.

[6] J. Szmidt, J. Urbanowicz and D. Zagier, Congruences among generalized Bernoulli numbers, Acta Arith. 71 (1995), 273-278.

[7] J. Urbanowicz and K. S. Williams, Congruences for L-functions, Math. Appl. 511, Kluwer, 2000.

Department of Mathematics \& Computer Science

Tsuda College

2-1-1 Tsuda-cho Kodaira-shi

Tokyo, 187-8577 Japan

E-mail: ota@tsuda.ac.jp

Received on 7.5.2001

and in revised form on 23.9.2002 